\title{
APLICAÇÃO DE GPR NO ESTUDO DE SUCESSÕES FLUVIAIS QUATERNÁRIAS NO MEGALEQUE DO TAQUARI, BACIA DO PANTANAL
}

\section{Rodrigo Diego Derubeis}

Dissertação apresentada ao Programa de Geociências Geoquímica e Geotectônica no Instituto de Geociências da Universidade de São Paulo para a obtenção do título de Mestre em Ciências.

Área de concentração: Geotectônica

Orientador: Professor Doutor Renato Paes de Almeida 
Autorizo a reprodução e divulgação total ou parcial deste trabalho, por qualquer meio convencional ou eletrônico, para fins de estudo e pesquisa, desde que citada a fonte.

Serviço de Biblioteca e Documentação do IGc/USP

Ficha catalográfica gerada automaticamente com dados fornecidos pelo(a) autor(a) via programa desenvolvido pela Seção Técnica de Informática do ICMC/USP

Bibliotecários responsáveis pela estrutura de catalogação da publicação: Sonia Regina Yole Guerra - CRB-8/4208 | Anderson de Santana - CRB-8/6658

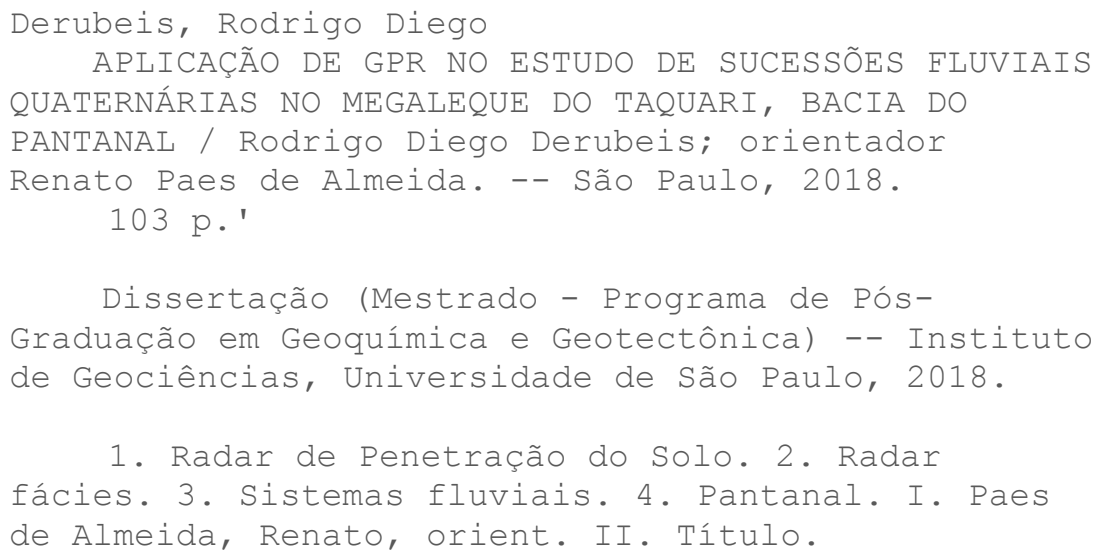




\title{
APLICAÇÃO DE GPR NO ESTUDO DE SUCESSÕES FLUVIAIS QUATERNÁRIAS NO MEGALEQUE DO TAQUARI, BACIA DO PANTANAL
}

\author{
RODRIGO DIEGO DERUBEIS
}

Orientador: Prof. Dr. Renato Paes de Almeida

Dissertação de Mestrado

$\mathrm{N}^{\circ} 809$

COMISSÃO JULGADORA

Dr. Renato Paes de Almeida

Dr. Vagner Roberto Elis

Dra. Milene Fornari

Dr. Vinicius Hector Abud Louro

São Paulo - Brasil

2018 
A minha esposa Vanessa, pelo apoio e amor durante este processo, e ao meu filho Raul Francisco, que veio para nos alegrar! 


\section{Agradecimentos}

À CAPES, Coordenação de Aperfeiçoamento de Pessoal de Nível Superior, e à FAPESP, Fundação de Amparo à Pesquisa do Estado de São Paulo, pelos suportes financeiros que viabilizaram esta pesquisa.

À Universidade de São Paulo, ao Instituto de Geociências, ao Programa de PósGraduação em Geoquímica e Geotectônica e aos seus funcionários, por possibilitarem a pesquisa. Ao Samuca, pelo apoio técnico na aquisição dos dados de campo.

Ao professor orientador Renato Paes de Almeida, pelos ensinamentos, apoio, confiança, orientação e atenção.

Aos professores Fábio Taioli, Maurício Guerreiro dos Santos e Mario Luis Assine, pelos ensinamentos, indicações e apoio.

Aos amigos da pós-graduação orientandos do professor Renato, pelo apoio, amizade e troca de conhecimentos em vários momentos, em especial Larissa Tamura e Ariel.

A minha família, pelo amor, amizade, incentivo e companheirismo: Vanessa, Maria Isabel, Alex, Luana, Fábio, Alessandra e Tenório. Aos meus amigos e amigas, pela amizade, incentivo, companheirismo e auxílios: Wesley, Requejo, Lívia, Fernanda, Bruno, Neto e Sumida. 


\section{Resumo}

O Pantanal é uma área mundialmente conhecida por ser uma das maiores planícies com alagamentos sazonais do mundo. Do ponto de vista de seus sistemas deposicionais, é uma área importante na caracterização de sistemas fluviais distributivos, característicos de áreas subsidentes e com grande relevância na interpretação do registro estratigráfico de sistemas fluviais em bacias sedimentares. Entretanto, os aspectos temporais da evolução dos sistemas fluviais do Pantanal são ainda pouco investigados, sendo ainda mais escassos os estudos com abordagem de investigações de sua subsuperfície. Como forma de contribuir para a compreensão da evolução temporal dos sistemas fluviais na região, o presente estudo apresenta os resultados de levantamentos de Radar de Penetração do Solo (GPR - Ground Penetrating Radar) e interpretação de radar fácies no Megaleque do Rio Taquari, no lobo meandrante do rio de mesmo nome, no Pantanal. Como resultado, após o processamento das informações coletadas, foram selecionadas nove seções GPR representativas do sistema, nas quais foram identificadas três radar fácies. Através da interpretação destas, foi possível identificar geometrias deposicionais em profundidades distintas do padrão formado pelos sistemas atuais, interpretadas como um sistema entrelaçado possivelmente de maior vazão que o atual e que implicam em transformações no sistema fluvial do rio Taquari no Quaternário, possivelmente devido a mudanças climáticas.

Palavras chaves: Radar de Penetração do Solo; Radar fácies; Sistemas fluviais; Pantanal. 


\begin{abstract}
The Pantanal is internationally recognized as one of the largest seasonally flooded areas in the world. From a depositional systems point of view, it is important in the characterization of distributive fluvial systems, typical of subsiding areas and bearing great relevance for the interpretation of the fluvial rock record preserved within sedimentary basins. Despite that, the temporal aspects of the evolution of the fluvial systems in Pantanal are still poorly investigated, and studies based on subsurface surveys are even more scarce. As a means to contribute for the understanding of the fluvial systems evolution in the region, this dissertation presents the results of Ground Penetrating Radar (GPR ) surveys and radar facies interpretation in the Taquari river megafan, specifically in the meandering lobe of the homonymous river in the Pantanal. After data processing, nine GPR sections representative of the system were selected, in which three radar facies were identified. Their interpretation lead to the identification of subsurface depositional geometries which are distinct from the modern day patterns, interpreted as a braided system possibly with greater discharge than the modern meandering river. This implies in changes in the Taquari fluvial system in the Quaternary, probably due to climate change.
\end{abstract}

Keywords: Ground Penetrating Radar; Radar facies; Fluvial systems; Pantanal 


\section{Sumário}

\section{Lista de Figuras}

\section{Lista de Tabelas}

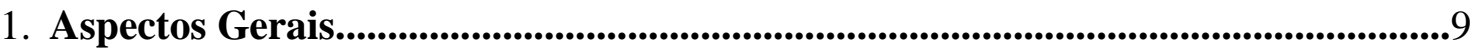

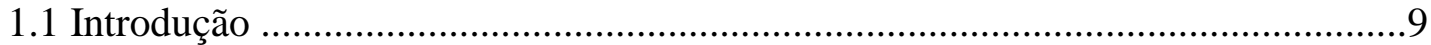

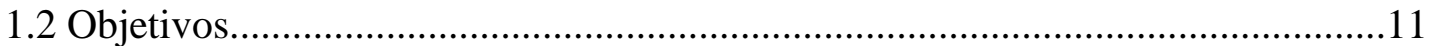

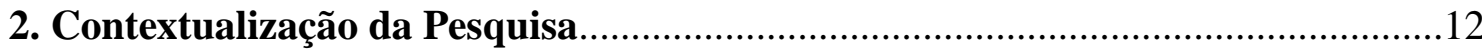

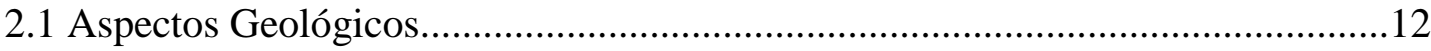

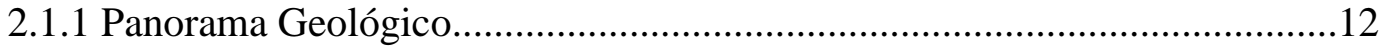

2.1.2 Formação da Bacia do Pantanal.....................................................................13

2.1.3 Bacia Sedimentar do Pantanal..................................................................... 15

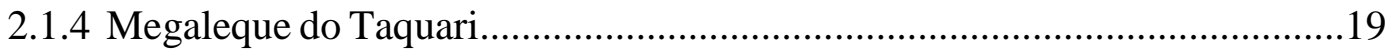

2.2 Radar de Penetração do Solo (GPR - Ground Penetrating Radar).......................24

2.2.1 Contextualização do Método GPR.................................................................24

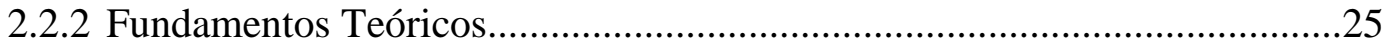

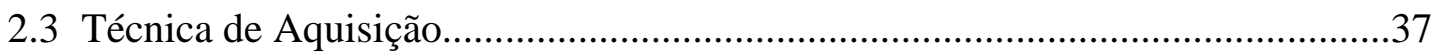

2.4 Revisão de Estudos em Ambientes Fluviais e Aluvial Utilizando o Método GPR.

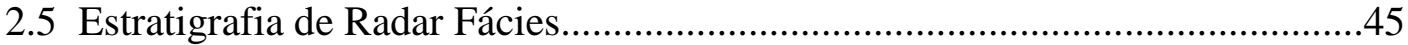

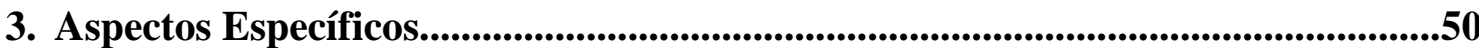

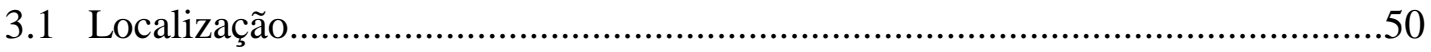

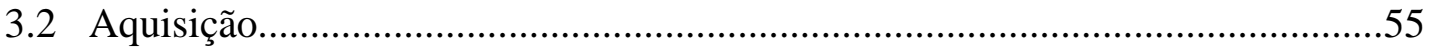

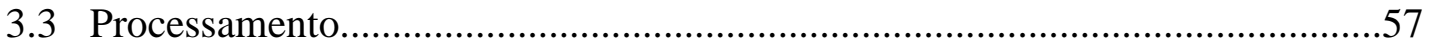


4. Resultados..

60

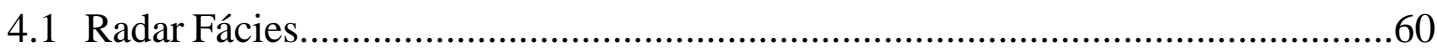

4.2 Descrição e Interpretação das Seções..............................................................64 a

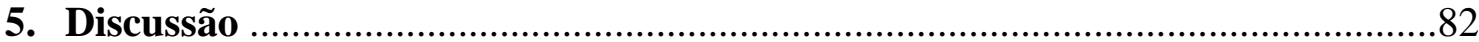

5.1 Comparação com Estudos Realizados no Rio Taquari e em Outros Rios..............82

5.2 Possíveis Mudanças Climáticas Responsáveis Pelas Alterações do Ambiente

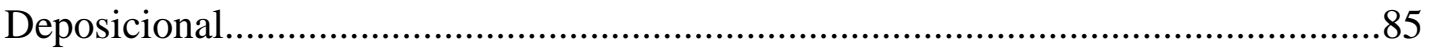

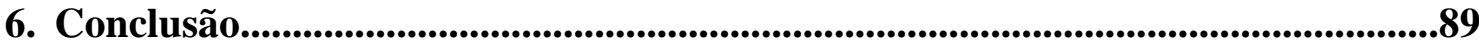

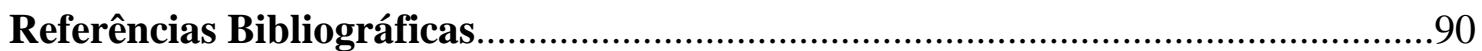




\section{Lista de Figuras}

Figura 2.1.1-1: Localização do Pantanal (Assine e Soares, 2004), com destaque para a região com altitude inferior a 200 m e a porção brasileira do Pantanal; bloco diagrama da Depressão do Alto Paraguai (Assine, 2010), com destaque para altitude inferior a $200 \mathrm{~m}$ (cores: cinza, azul e verde claro).

Figura 2.1.2-1: Modelo da evolução geológica do Pantanal, início da bacia durante o Neógeno (Assine et al., 2016).....

Figura 2.1.2-2: Modelo da evolução geológica do Pantanal, início da bacia durante o Paleógeno (Assine et al., 2016)

Figura 2.1.3-1: Mapa Geológico. Unidades cronoestratigráficas: $\mathrm{M}=$ Meso-Proterozóico, $\mathrm{N}=$ Neo-Proterozóico, $\mathrm{O}=$ Ordoviciano, $\mathrm{S}=$ Siluriano, $\mathrm{D}=$ Devoniano, $\mathrm{C}=$ Carbonífero, $\mathrm{P}=$ Permiano, $\mathrm{J}=\mathrm{Jurássico,} \mathrm{K}=$ Cretáceo, $\mathrm{T}=$ Terciário, $\mathrm{Q}=$ Quaternário. Planaltos: 1 = Bodoquena; 2 = Maracajú-Campo Grande; 3 = Taquari-Itiquira; 4 = Guimarães; 5 = Parecis; 6 = Província Serrana; 7 = Urucum-Amolar (Assine, 2003) 16

Figura 2.1.3-2: Espessura da Formação Pantanal (modificado de Assine, 2003). .17

Figura 2.1.3-3: Lineamento Transbrasiliano (Assine, 2003) .18

Figura 2.1.4-1: Megaleques fluviais que compõem o trato deposicional do Pantanal: 1= Taquari, 2= Cuiabá, 3= São Lourenço, 4 = Corixo Grande, 5 =Taboco, 6 =Aquidauana e 7 =Nabileque (modificado de Zani, 2008)....

Figura 2.1.4-2: Megaleque do Rio Taquari indicando curvas de nível, paleocanias, cinturão de meandramento, lobos atuais e lobos abandonados. Perfis topográficos de leques aluviais (modificado de Assine, 2003).

Figura 2.1.4-3: Divisão do Pantanal em sub-regiões (Padovani, 2010) .22

Figura 2.2.2-1: Decaimento da amplitude modificado de Annan( 2001) .30 
Figura 2.2.2-2: Fatores que contribuem para as perdas de energia da onda eletromagnética (Reynolds, 1997)

Figura 2.2.2-3: Largura da radiação da frente de onda da primeira zona de Fresnel (Reynolds, 1997) 35

Figura 2.2.2-4: Largura da radiação da frente de onda da primeira zona de Fresnel (Neal, 2004)

Figura 2.3-1: Esboço esquemático da aquisição de dados, mostrando componentes do sistema GPR e a configuração do refletor subsuperficial (Neal, 2004) 37

Figura 2.3-2: a - Radargrama resultante da plotagem sequencial de traços individuais de pontos de levantamento adjacentes. São indicados a onda aérea, a onda direta do solo e as reflexões primárias. $\mathrm{b}$ - Percursos da onda entre as antenas transmissora e receptora, para a onda de ar, a onda do solo, a onda lateral e a onda refletida (modificado de Neal, 2004)........38

Figura 2.3-3: a - Diagrama da aquisição Common Offset. b - Diagrama da aquisição WARR (Neal, 2004) 39

Figura 2.3-4: Radargrama de uma sondagem de veocidades feita com antenas de $100 \mathrm{MHz}$ (Huisman, 2003)

Figura 2.3-5: Percursos de propagação das ondas eletromagnéticas em um solo com duas camadas de permissividade dielétrica contrastante, sendo $\varepsilon 1>\varepsilon$ em uma sondagem de veocidades WARR, indicando as ondas aéreas, diretas do solo, refratadas, refletidas e refratadas criticamente (Huisman, 2003)

Figura 2.3-6: Diagrama esquemático de reflexões e refrações de uma sondagem de velocidades WARR. A onda no solo pode ser identificada como uma onda com um movimento linear a partir da origem do gráfico x - t. Nas equações de declive, c é a velocidade eletromagnética no ar e x é a separação da antena (Huisman, 2003) .41 
Figura 2.4-1: Os sete padrões de radar fácies acima e uma seção GPR esquemática com as radar fácies (Franke et al. 2015).

Figura 2.5-1: Terminologia para definir e descrever superfícies de radar, pacotes de radar e fácies de radar (Neal, 2004).

Figura 2.5-2: Classificação da hierarquia de superfícies limitantes. O diagrama superior mostra um canal com vários grandes depósitos de macroformação. O diagrama central mostra a estratificação em uma macroforma, incluindo as superfícies de acreção, e o diagrama inferior mostra detalhes dentro dessa estratificação (Miall, 1988).

Figura 3.1-1: Imagem com a Bacia do Alto Paraguai em verde escuro, o Pantanal em verde médio e o Megaleque do Rio Taquari em verde claro (imagem modificada da internet: http://www.riosvivos.org.br/arquivos/site_noticias_927317926.jpg, acessado em fevereiro de 2018). 50

Figura 3.1-2: Imagem indicando o Megaleque do Rio Taquari em verde claro, o local onde foram realizadas as aquisições em laranja, o Rio Taquari em lilás e os municípios de Corumbá em vermelho e de Coxim em amarelo

Figura 3.1-3: Imagem da região onde foram realizadas as aquisições (ampliação da região laranja da imagem 3.1-2), com destaque para as regiões onde foram realizadas as aquisições em branco, verde claro e amarelo. Indicação do local das seções GPR com setas laranjas, indicação do local das seções WARR e trado com estrelas vermelhas e indicação das estradas locais em amarelo. .52

Figura 3.1-4: Imagem da região onde foram realizadas as aquisições (ampliação da região branca da imagem 3.1-3), indicação do local das seções GPR com setas laranjas, indicação do local das seções WARR e trado com estrelas vermelhas e estradas locais em amarelo. .53

Figura 3.1-5: Imagem da região onde foram realizadas as aquisições (ampliação da região azul da imagem 3.1-3), com indicação do local das seções GPR com setas laranjas, indicação do local das seções WARR e trado com estrelas vermelhas e estradas locais em amarelo........53 
Figura 3.1-6: Imagem da região onde foram realizadas as aquisições (ampliação da região amarela da imagem 3.1-3), com indicação do local das seções GPR com setas laranjas, indicação do local das seções WARR e trado com estrelas vermelhas e estradas locais em amarelo .54

Figura 3.2-1 - Antenas transmissora e receptora da IDS de 100MHz, utilizadas neste estudo.55

Figura 3.2-2: Imagens de aquisições realizadas em campo e indicações dos equipamentos que constituem o método GPR: Antenas, odômetro, unidade de controle (DAD) e notebook .56

Figura 4.2-1:a) Detalhe da localização da seção. b) Imagem da seção A, com detalhes de quatro trechos e suas respectivas radar fácies .65

Figura 4.2-2:a) Detalhe da localização da seção. b) Imagem da seção B, com detalhes de três trechos e suas respectivas radar fácies

Figura 4.2-3:a) Detalhe da localização da seção. b) Imagem da seção G, com detalhes de dois trechos e suas respectivas radar fácies

Figura 4.2-4:a) Detalhe da localização da seção. b) Imagem da seção H, com detalhe de um trecho e suas respectivas radar fácies .71

Figura 4.2-5:a) Detalhe da localização da seção. b) Imagem da seção C, com detalhe de um trecho e suas respectivas radar fácies .73

Figura 4.2-6:a) Detalhe da localização da seção. b) Imagem da seção D, com detalhes de três trechos e suas respectivas radar fácies .75

Figura 4.2-7:a) Detalhe da localização da seção. b) Imagem da seção E, com detalhes de três trechos e suas respectivas radar fácies .77

Figura 4.2-8:a) Detalhe da localização da seção. b) Imagem da seção F, com detalhes de dois trechos e suas respectivas radar fácies .79 
Figura 4.2-9:a) Detalhe da localização da seção. b) Imagem da seção F, com detalhes de quatro trechos e suas respectivas radar fácies.

Figura 5.2-1: Áreas onde foram obtidos os testemunhos de sondagem em preto e local dos dados deste trabalho em vermelho (modificado de Assine, 2013).

Figura 5.2-2: Gráfico das concentrações de isótopos por milhares de anos (modificado de (Wang et al., 2017)

Figura 5.2-3:imagem com destaque para Zona de Convergência Intertropical (ITCZ), Zona de Convergência da América do Sul (SACZ), A seta branca indica a trajetória geral simplificada do Jato de Baixo Nível (LLJ) (modificado de Bernal et al., 2016). 


\section{Lista de Tabelas}

Tabela 2.1.4-1:Vazões mensais e anuais das estações Coxim e Porto Rolon (A.N.A., 2004)..21

Tabela 2.2.2-1: Exemplos de valores das propriedades elétricas (Neal, 2004).

Tabela 2.2.2-2: Frequência e profundidade de penetração (Porsani, 1999)

Tabela 2.2.2-3: Frequência e Resolução vertical (Porsani, 1999).

Tabela 4.1-1: Padrões de radar fácies, organizados em código, descrição, interpretação e imagem de cada tipo de radar. 


\section{Aspectos Gerais}

\subsection{Introdução}

Esta pesquisa teve o apoio da CAPES com a concessão de bolsa de mestrado. A aquisição dos dados geofísicos de GPR foi realizada por uma equipe composta por: Mestrando Rodrigo Diego Derubeis (Instituto de Geociências - IGc - USP), Técnico Samuel Pedro Egídio (Samuca), Prof. Dr. Mauricio Guereiro (Centro de Engenharia, Modelagem e Ciências Sociais Aplicadas - CECS , Universidade Federal do ABC - UFABC) e Prof. Dr. Fábio Taioli (Instituto de Geociências - IGc - USP), sendo este último o responsável pela aquisição, que foi financiada pelo projeto FAPESP 2014/0688992 denominado "Mudanças paleo-hidrológicas, cronologia de eventos e dinâmica sedimentar no Quaternário da Bacia do Pantanal", em desenvolvimento desde 2014 e coordenado pelo Prof. Dr. Mario Luis Assine (Instituto de Geociências e Ciências Exatas - Universidade Estadual Paulista UNESP - Rio Claro).

A pesquisa trata da identificação de geometrias deposicionais na Bacia do Pantanal no Quaternário, mais especificamente no Megaleque do Rio Taquari, com a utilização de Radar de Penetração do Solo (GPR - Ground Penetrating Radar) em sucessões sotopostas aos depósitos do sistema fluvial ativo, e das implicações para a evolução desse sistema.

Sob o ponto de vista geológico, o Pantanal é uma ampla área subsidente (Ussami, 1999) na qual predominam sistemas deposicionais fluviais da Bacia do Rio Paraguai, incluindo amplas planícies de inundação e cinturões de canais dos principais rios. Nessa área, é marcante a presença de megaleques aluviais dos principais tributários do Rio Paraguai, destacando-se os rios São Lourenço e Taquari. Assim, a região compõe uma das maiores planícies com alagamentos sazonais do mundo, com amplas planícies inundáveis, presença de lagos permanentes e temporários e ciclos de cheias periodicamente regulares.

Os sistemas fluviais do Pantanal são altamente dinâmicos, registrando mudanças ambientais nas últimas décadas (Assine, 2003), como as alterações dos cursos dos rios e a variação da vazão. No caso específico do Rio Taquari, onde foram coletados os dados deste estudo, também observa-se, em um período recente, alterações ao longo de seu curso e variações de sua vazão, que possui média anual no período de 1966 a 2003 de 320 m³/s, na 
estação fluviométrica de Coxim localizada na cidade homônima, próxima de onde foram coletado os dados. Essa dinâmica relaciona-se aos padrões radiais de canais reliquiares e ativos encontrados nos megaleques. Contudo, a dinâmica histórica pode não refletir os padrões de variação em escala de tempo mais longa.

Os aspectos temporais da evolução desses sistemas ao longo do Quaternário são ainda pouco investigados e são particularmente raros os estudos com abordagem de investigações de sua subsuperfície. Como forma de contribuir para a compreensão da evolução temporal dos sistemas fluviais na região, o presente estudo apresenta os resultados de levantamentos de Radar de Penetração do Solo (GPR - Ground Penetrating Radar) e interpretação de radar fácies no Megaleque do Rio Taquari, no lobo meandrante do rio de mesmo nome, no Pantanal, com o objetivo de identificar geometrias deposicionais em profundidade e de testar a hipótese de variações significativas nos padrões de canais e barras ao longo do tempo geológico.

A presente dissertação está organizada em seis capítulos: o primeiro deles, intitulado “Aspectos Gerais", apresenta uma breve introdução da pesquisa, com informações sobre a sua realização, como o local de desenvolvimento, a justificativa, o método empregado e os objetivos; o segundo capítulo, "Contextualização da Pesquisa", aborda a geologia da área estudada, os procedimentos específicos do método empregado, a importância de outras pesquisas já realizadas com o mesmo método neste ambiente ou em ambientes semelhantes e a metodologia utilizada para a interpretação dos dados obtidos; no terceiro capítulo, "Aspectos Específicos", foram detalhados os locais de aquisição dos dados e a forma como foram obtidas as informações que posteriormente foram processadas com o software selecionado. No capítulo quatro, denominado "Resultados", são apresentadas as radar fácies identificadas, suas interpretações e as seções obtidas com o método empregado, processadas e interpretadas. No quinto capítulo, "Discussão", são apresentadas as análises e interpretação dos dados em termos das características dos sistemas responsáveis pelos elementos arquiteturais e limites litológicos identificados no quarto capítulo, enfatizando as diferenças entre os depósitos antigos e os sistemas atuais. Por fim, no sexto capítulo, "Conclusão", são sintetizados os dados e interpretações. 


\subsection{Objetivos}

A presente dissertação tem como objetivo principal investigar as estruturas sedimentares preservadas em subsuperfície em áreas selecionadas do Megaleque do Rio Taquari, afluente da margem esquerda do Rio Paraguai na região do Pantanal. Buscou-se testar a hipótese de modificações significativas nos estilos de canais e na arquitetura deposicional em decorrência das modificações climáticas do fim do Pleistoceno e do Holoceno. Para tanto, os objetivos parciais foram:

1) Testar a eficácia da investigação geofísica de radar de penetração do solo (Ground Penetrating Radar - GPR) na interpretação de geometrias deposicionais em subsuperfície no Pantanal, dada a grande variabilidade de resposta desse método em função das características do substrato.

2) Confirmada a eficiência do método na região, identificar estruturas sedimentares, elementos arquiteturais e limites litológicos, com base em interpretações de radar fácies, comparando três regiões de investigação e suas evoluções ao longo do tempo. 


\section{Conclusão}

O presente estudo foi proposto para investigar a região do Pantanal, mais especificamente o lobo meandrante do Rio Taquari, no Megaleque de mesmo nome. A metodologia utilizada, o Ground Penetrating Radar - GPR, mostrou-se eficaz na tarefa de imagear a subsuperfície, sendo possível observar estruturas em uma profundidade próxima a $10 \mathrm{~m}$, ou seja, podemos afirmar que o método GPR se mostrou eficaz neste estudo do Pantanal.

Foi possível identificar três radar fácies, denominadas R1, R2 e R3. R1 foi interpretada como limites contínuos de séries de laminação cruzada, que por suas dimensões não permitiram a observação de estratos cruzados dentro da resolução, e de níveis contínuos de depósitos de decantação. Provavelmente, tratam-se de depósitos de planície de inundação. R2 foi interpretada como barras, originadas com a deposição de sedimentos nas curvas do rio e o acúmulo dos sedimentos trazidos pelo fluxo do rio nas margens convexas. R3 foi interpretada como estratificações cruzadas acanaladas de dunas de médio a pequeno porte.

Outro intuito deste trabalho foi o de observar possíveis diferenças entres as três regiões investigadas, porém não foi observada nenhuma variação significativa nos padrões de refletores, o que pode ser explicado devido à pequena distância entre essas três regiões.

Foram observadas variações verticais de radar fácies em praticamente todas as seções. $\mathrm{R} 1$, a mais superficial, sendo-lhe atribuídas características de mais recente, e R3 a mais profunda e mais antiga. Estas variações na distribuição das radar fácies foram interpretadas como alterações de ambiente deposicional e as prováveis causas dessas alterações podem ser as mudanças climáticas ocorridas no passado, em escala global e regional, com a passagem de depósitos de rios de maior vazão, talvez entrelaçados, formados em um dos ciclos de maior precipitação do fim do Pleistoceno, passando a sistemas com características semelhantes aos atuais rios meandrantes. 


\section{Referências Bibliográficas}

Ab' Sáber, A. N., 1988. O Pantanal Mato-Grossense e a teoria dos refúgios. Revista Brasileira de Geografia 50: 9-57

Ab' Sáber, A. N., 2006. Brasil: Paisagens de Exceção: o litoral e o Pantanal Mato Grossense: patrimônios básicos. Cotia - SP: Ateliê Editorial. p. 11-78

A.N.A., 2004. Implementação de Práticas de Gerenciamento Integrado de Bacia Hidrográfica para o Pantanal e Bacia do Alto Paraguai ANA/GEF/PNUMA/OEA: Programa de ANA ... [et al .]. Ações Estratégicas para o Gerenciamento Integrado do Pantanal e Bacia do Alto Paraguai: Relatório Final/Agência Nacional de Águas — Brasília: TDA Desenho \& Arte Ltda., p. 29-57

Annan, A.P., 2001. Ground Penetrating Radar: Workshop Notes: Sensors e Software, Inc., p. 192.

Assine, M. L., 2003. Sedimentação na Bacia do Pantanal Mato-Grossense, Centro-Oeste do Brasil. Tese de Livre-Docência, Universidade Estadual Paulista - UNESP, Rio Claro. p. 106.

Assine, M. L., 2009. Taquari: um rio mutante. In: Anais $2^{\circ}$ Simpósio de Geotecnologias no Pantanal, Corumbá, Embrapa Informática Agropecuária/INPE, p.1034-1040.

Assine, M. L., 2010. Pantanal Mato-Grossense: uma paisagem de exceção. In: (ed.) A Obra de Aziz Nacib Ab'Sáber. p. 464 - 489. 
Assine M.L. and Soares P.C., 2004. Quaternary of the Pantanal, west-central Brazil. Quaternary International, 114:23-34.

Assine M.L., Merino E.R., Pupim F.N., Macedo H.A., Santos M.G.M., 2015. The Quaternary alluvial systems tract of the Pantanal Basin, Brazil. Brazilian Journal of Geology,45(3):475-489

Assine, M.L., Merino, E.R., Pupim, F.N., Warren, L.V., Guerreiro, R.L., McGlue, M.M., 2016. Geology and geomorphology of the Pantanal Basin. In: Bergier, I., Assine, M.L. (Eds.), Dynamics of the Pantanal Wetland in South America. Springer International, Cham, Switzerland, p. 23-50.

Bernal J.P., Cruz F.W., Strikis N.M., Wang X., Deininger M., Catunda M.C.A., OrtegaObregon C., (...), Auler A.S. ,2016. High-resolution Holocene South American monsoon history recorded by a speleothem from Botuverá Cave, Brazil. Earth and Planetary Science Letters, 450 , pp. 186-196

Bridge, J. S., 2003. Rivers and Floodplains: Forms, Processes and Sedimentary Record, 491 pp., Blackwell, Malden, Mass.

Cassidy N.J., 2009. Electrical and Magnetic Properties of Rocks, Soils and Fluids, in Jon, H. M. org., Ground Penetrating Radar Theory and Applications, Elsevier B.V., p. 41-67

Daniels, D. J., 2004. Ground Penetrating Radar, 2nd Edition, : Radar, sonar and avionics, London, Institution of Electrical Engineers, p. 1 e 11

Franke, D., Hornung, J., Hinderer, M., 2015. A combined study of radar facies, lithofacies and three-dimensional architecture of an alpine alluvial fan (Illgraben fan, Switzerland). Sedimentology 62, 57-86. 
Huisman, J. A., Hubbard, S. S., Redman, J. D. and Annan, A. P.,2003. Measuring soil water content with ground penetrating radar: A review, Vadose Zone J., 2: 476-491.

Jol, H. M., and Bristow, C. S.,2003. GPR in sediments: advice on data collection, basic processing and interpretation, a good practice guide. Geological Society, London, Special Publications, 211(1), p. 9-27

LeClair, S.F. and Bridge, J.S., 2001. Quantitative interpretation of sedimentary structures formed by river dunes. J. Sed. Res., 71, 713-716.

Lopes M., Fornari, M., Taioli F. 2013. Characterization of the architecture of fluvial bars of the Amazon and Tapajós rivers using GPR (ground penetrating radar). In: 13rd International Congress of the Brazilian Geophysical Society. p. 7.

Miall, A.D., 1988. Architectural elements and bounding surfaces in fluvial deposits: Anatomy of the Kayenta Formation (Lower Jurassic), southwest Colorado. Sediment. Geol., 55: 233-262,

Miall, A.D., 1996. The Geology of Fluvial Deposits: Sedimentary Facies, Basin Analysis and Petroleum Geology. New York, Springer-Verlag, p. 582

Neal, A.,2004. Ground-penetrating radar and its use in sedimentology: Principles, problems and progress: Earth-Science Reviews, v. 66, p. 261-330

Olhoeft, G. R.,2002. Applications and Frustrations in Using Ground Penetrating Radar, Colorado School of Mines, IEEE AESS Svstems Mugazinr, p. 12-20.

Padovani C. V., 2010. Dinâmica das Inundações do Pantanal. Tese de Doutorado. Universidade de São Paulo - USP/ESALQ. Piracicaba -SP. p. 174. 
Paola, C. and Borgman, L., 1991. Reconstructing random topography from preserved stratifcation, Sedimentology, 38, 553-565

Porsani J.L., 1999. Ground Penetrating Radar (GPR): Proposta Metodológica de Emprego em Estudos Geológico-Geotécnicos nas Regiões de Rio Claro e Descalvado - SP, Tese de Doutorado. Instituto de Geociências e Ciências Exatas, Universidade Estadual Paulista Júlio de Mesquita (UNESP), Rio Claro.

Porsani, J.L.; Assine, M.L. ; Moutinho, L. , 2005. Application of GPR in the Study of a Modern Alluvial Megafan: the Case of the Taquari River in Pantanal Wetland, WestCentral Brazil. Subsurface Sensing Technology and Applications, v. 6, n.2, p. 219233.

Pupim F.N. 2014.Geomorfologia e paleo-hidrologia dos megaleques dos rios Cuiabá e São Lourenço, Quaternário da Bacia do Pantanal MS Tese de Doutorado, Universidade Estadual Paulista - UNESP, Rio Claro - SP, 109p.

Reynolds, J.M., 1997. An Introduction to Applied and Environmental Geophysics. Wiley, Chichester., p. 681-777

Sambrook Smith, G.H., Ashworth, P.J., Best, J.L. Woddward, J., Simpson, G.J., 2006. The sedimentology and alluvial architecture of sandy braided South Saskatchewan river, Canada. Sedimentology, 53, p. 413-434.

Sambrook Smith, G.H., Ashworth, P.J., Best, J.L., Lunt, I.A.,Orfeo, O, Parsons, D.R., 2009. The sedimentology and alluvial architecture of a large braid bar, Rio Paraná, Argentina. Journal of Sedimentary Research, 79, p. 629-642 
Souza, L.A.P. ; Porsani, J.L. ; Souza, O.C. ; Moutinho, L., 2002. Levantamento Experimental GPR no Rio Taquari, Bacia do Pantanal Matogrossense. Revista Brasileira de Geofísica, Rio de Janeiro, v. 20, n.1, p. 67-72.

Ussami, N.; Shiraiwa, S.; Dominguez, J. M. L., 1999. Basement reactivation in a sub-Andean foreland flexural bulge: The Pantanal wetland, SW Brazil. Tectonics, 18: 25-39.

Vandenberghe J, and van Overmeeren RA. 1999. Ground penetrating radar images of selected fluvial deposits in the Netherlands. Sedimentary Geology 128: 245-270.

Wang, X., Edwards, R.L., Auler, A.S., Cheng, H., Kong, X., Wang, Y., Cruz, F.W., Dorale, J.A., Chiang, H.-W., 2017. Hydroclimate changes across the Amazon lowlands over the past 45,000 years. Nature 541:204-207.

Zani H. 2008. Mudanças morfológicas na evolução do megaleque do Taquari: uma análise com base em dados orbitais. Dissertação de Mestrado, Universidade Estadual Paulista - UNESP, Rio Claro. p. 85. 